\title{
Parsing HL7 aECG Files and Segmenting Leads for Interactive Progressive-based Interpretation of the 12-lead Electrocardiogram
}

\author{
Andrew W Cairns ${ }^{1}$, Raymond R Bond ${ }^{1}$, Dewar D Finlay ${ }^{1}$, Daniel Guldenring ${ }^{1}$, Fabio Badilini ${ }^{2}$, \\ Guido Libretti ${ }^{2}$, Aaron J Peace ${ }^{3}$ \\ ${ }^{1}$ Ulster University, Northern Ireland, UK, ${ }^{2}$ AMPS-LLC, ${ }^{3}$ Altnagelvin Hospital, Northern Ireland, UK
}

\begin{abstract}
The 12-lead Electrocardiogram (ECG) is ubiquitously used as a diagnostic support tool to detect cardiovascular disease. Nevertheless, it is often difficult to read and incorrectly interpreted due to the significant cognitive load forced upon the interpreter. However, ECG interpretation errors can be reduced through utilizing interactive touch screen devices which facilitate a systematic approach to aid ECG interpretation. In view of this, a set of interactive questions and prompts to guide an interpreter through a typical ECG reporting process had been developed and coined 'Interactive Progressive based Interpretation' (IPI).

To realise the potential of this system a pathway for potential interpreters must be created. Often, an ECG is stored in XML format. Therefore, to allow practitioners to unobtrusively use the IPI system it must be capable of consuming this file format, and process its data.

To achieve this, we have partnered with AMPS-LLC to create a model which can consume an HL7-XML file, and converts it into specific segmented image files in a desired format (PDF, PNG or JPG). Once these images are segmented, they can then be placed automatically into the IPI system sequence.

In conclusion, a pathway for a decision support model has been created to aid ECG interpretation. We hypothesize this could facilitate a diagnostic aid in ECG interpretation.
\end{abstract}

\section{Introduction}

With the prevalence of Cardiovascular Disease (CVD), contributing to $29 \mid \%$ of worldwide fatalities, it is imperative to optimize its early detection. Although the 12lead Electrocardiogram (ECG) has been the primary method of assessing the cardiac state of a patient for more than 70 years, by the early 1960 s computer decision support began to be introduced to augment human interpretation [1], [2]. As time progressed, algorithms became more sophisticated [3] and commercially available [4]-[6].

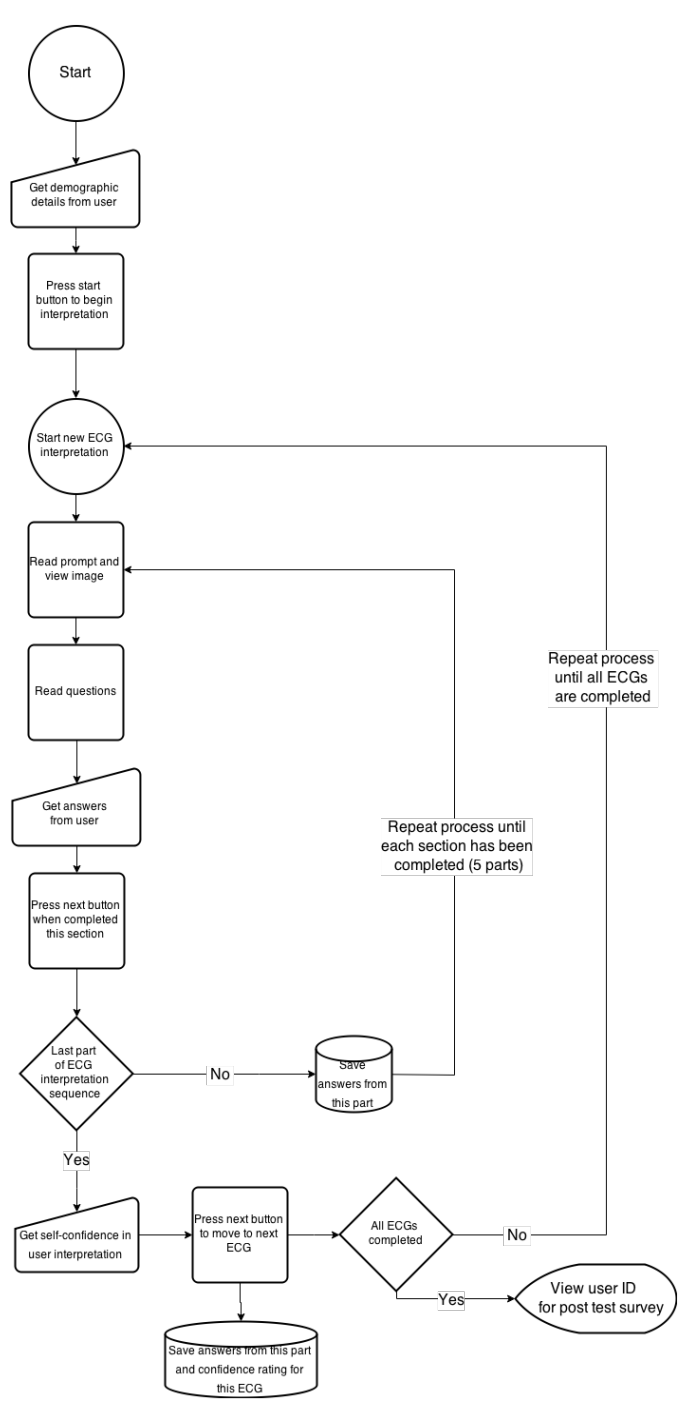

Figure 1. Procedural sequence for interpreters using the IPI model and system to interpret a 12-lead ECG. 
Typically, a 12-lead ECG is presented in a $3 \times 4+1 \mathrm{R}$ grid format with each cell representing one of the 12 leads and an extended rhythm strip added below $(+1 \mathrm{R})$ [7]. This format, is known to deliver significant cognitive load, and thereby deplete the cognitive performance of an interpreter [8]. To ameliorate cognitive workload, computerized decision support systems (CDSS) often aim to reduce the cognitive workload forced upon the interpreter [9]. To achieve this some institutions often use checklist in ECG reporting. [10][11][12][13][14][15][16]. Although some components vary, a typical reporting procedure would include; 1) heart rate, 2) rhythm analysis, 3) cardiac axis, 4) conduction times, 5) morphological features, and 6) final diagnoses. Other facilities have conducted research using a series of questions and prompts to guide the user though the interpretive process [17], [18]. This research also aimed to reduce the cognitive load by segmenting the 12-lead ECG into the five previously mentioned reporting components. Further motivations for this research include developing preventative measures to reduce the likelihood of 'early satisfaction syndrome' [19],[20], in which interpreters come to a conclusion prematurely. A model was created and named "Interactive Progressive based Interpretation" (IPI). The procedural sequence can be seen in Figure 1.

Results from this study indicate an increase in final interpretation accuracy can be achieved through using interactive touch screen devices to manage cognitive load alongside prompts to guide an interpretation process. Hence, a recommendation was suggested to incorporate a set of distinct procedural steps within the interpretation process, serving as a 'checklist' to eradicate missed coabnormalities [17].

\section{Methods}

Although a CDSS which manages an interpreters cognitive load has potential to improve diagnostic accuracy a pathway to practice needs to be established enabling a clinician/researcher to efficiently use the software. Typically, a 12-lead ECG is stored in Portable Document format (PDF) or in raw data within XML, SCPECG, DICOM, HL7-aECG, ecgML, Philips XML, mECGml, MFER or XML-ECG [21]. AMPS-LLC aims to create a paradigm which stores ECG data inside the commonly used PDF format [22]-[25].

To achieve this, AMPS-LLC extracts ECG recording data (often stored within an XML file), including; demographics, measures, interpretations, and morphologies among others. This data then populates a proprietary AMPS data structure. Once this is populated it is restructured into desired proprietary formats for use within the healthcare system (XML, DIACOM/MORTARA etc.). This is then embedded with a generated PDF. AMPS-LLC also provides the opportunity to output other graphical formats including PNGs or JPGs.
Due to the data acquisition and structural method each lead is stored individually within a data framework.

Therefore, potential to generate a segmented ECG for use within the IPI system became apparent. By partnering with AMPS-LLC a platform was created to upload an ECG recording data file (XML), a response was generated which produced multiple segmented ECG images, and hosted them on the Azure server. These images would then be placed within the IPI model, available for user interaction. This enables users (clinicians, researchers or others) to upload ECG data stored within healthcare repositories and automatically be presented with an interactive CDSS to aid the clinical decision making process. A figure for this pathway can be seen in Figure 2.

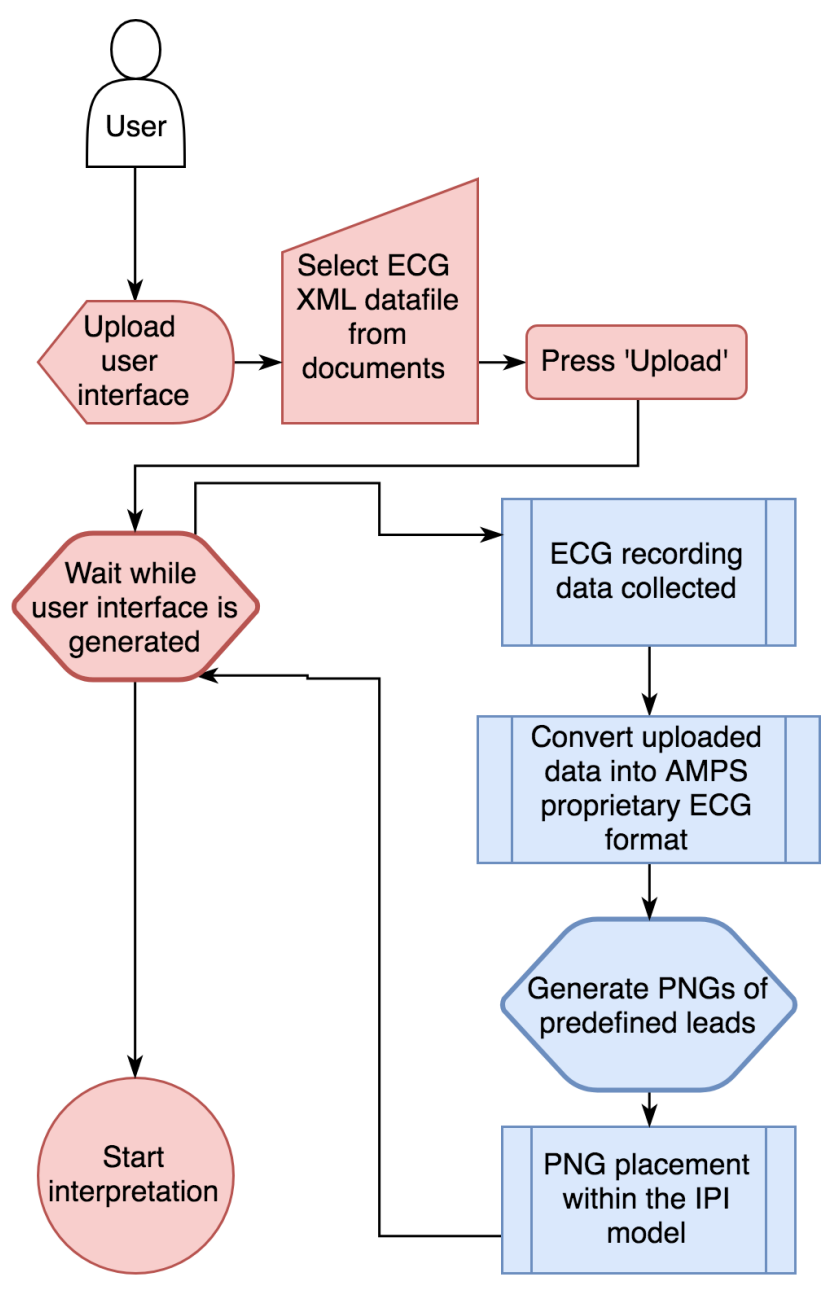

Figure 2. User flow and image generation procedure for IPI model to be used alongside AMPS-LLC

conversion tools to create a pathway with practice for ECG interpretation. 


\section{Model implementation}

To allow the system to be used ubiquitously it has been developed using web-based technologies and hosted on the university server. The webpage structure was created and implemented using Hypertext Mark-up Language version 5 (HTML5). Cascading Style Sheets (CSS3) were used to create a consistent, user, friendly, and responsive user experience for interpreters. The scripting language, JavaScript, was used, alongside the JQuery library, was used to create an interactive experience by implementing responsive animations and collection of data through text/button/radio/checkbox/slider field entry. Finally, the Hypertext Pre-processing language (PHP) recorded data entry values to a MySQL database. Data was sent to the server via Asynchronous JavaScript and XML (AJAX).

AMPS software is implemented using $\mathrm{C}++$ and hosted on a Microsoft Azure web server. The connection between this webserver and the front-end user interface was controlled asynchronously via AJAX. This enabled calls to request a PDF conversion, retrieve a JSON (JavaScript Object Notation) response with an embedded link to a series of PNGs hosted on the Azure webserver.

\section{Conclusion}

An interactive model has been developed to reduce cognitive workload forced upon interpreters of 12-lead ECGs. A potential 'pathway to practice' has now been created facilitating interpreters the opportunity to upload raw ECG files (XML) and have a bespoke user interface generated which integrates the uploaded ECG into the IPI model. We believe this example illustrates the potential to augment 12-lead ECG interpretation without needing to replace the conventional method.

\section{References}

[1] P. M. Rautaharju, "The birth of computerized electrocardiography: Hubert V. Pipberger (19201993).," Cardiol. J., vol. 14, no. 4, pp. 420-1, 2007.

[2] H. V PIPBERGER, R. J. ARMS, and F. W. STALLMANN, "Automatic screening of normal and abnormal electrocardiograms by means of digital electronic computer.," Proc. Soc. Exp. Biol. Med., vol. 106, pp. 130-2, Jan. 1961.

[3] P. W. MacFarlane, "A brief history of computerassisted electrocardiography," Methods of Information in Medicine, vol. 29, no. 4. pp. 272281, 1990.

[4] B. M. RuDusky and B. M. RuDusky, "Errors of
Computer Electrocardiography," Angiology, vol. 48, no. 12, pp. 1045-1050, Dec. 1997.
[5] J. L. Willems et al., "Evaluation of ECG interpretation results obtained by computer and cardiologists.," Methods Inf. Med., vol. 29, no. 4, pp. 308-16, Sep. 1990.

C. Abreu-Lima and J. P. de Sá, "Automatic classifiers for the interpretation of electrocardiograms.," Rev. Port. Cardiol., vol. 17, no. 5, pp. 415-28, May 1998.

J. E. Madias, "The 13th multiuse ECG lead: Shouldn't we use it more often, and on the same hard copy or computer screen, as the other 12 leads?," J. Electrocardiol., vol. 37, no. 4, pp. 285287, Oct. 2004.

M. Sibbald, A. B. H. de Bruin, and J. J. G. van Merrienboer, "Checklists improve experts' diagnostic decisions," Med. Educ., vol. 47, no. 3, pp. 301-308, 2013.

G. Q. Gao, "Computerised detection and classification of five cardiac conditions," 2003.

] C. J. Breen, R. Bond, and D. Finlay, "An evaluation of eye tracking technology in the assessment of 12 lead electrocardiography interpretation.," J. Electrocardiol., vol. 47, no. 6, pp. 922-929, Aug. 2014.

G. Wood, J. Batt, A. Appelboam, A. Harris, and M. R. Wilson, "Exploring the impact of expertise, clinical history, and visual search on electrocardiogram interpretation.," Med. Decis. Making, vol. 34, no. 1, pp. 75-83, Jan. 2014.

$\mathrm{R}$. Zeng et al., "New ideas for teaching electrocardiogram interpretation and improving classroom teaching content.," Adv. Med. Educ. Pract., vol. 6, pp. 99-104, Jan. 2015.

J. R. Hampton, The ECG Made Easy, Eighth. Elsevier Health Sciences, 2013.

J. H. O. Jr., S. C. Hammill, M. S. Freed, and S. M Pogwizd, The Complete Guide to ECGs. Jones \& Bartlett Publishers, 2010.

D. Dublin, "Dublin's Method for Reading EKG's," in Rapid interpretation of EKG's, Sixth., Cover Publishing Company, 2000, pp. 335-346.

T. Raupach, N. Hanneforth, S. Anders, T. Pukrop, O. Th J Ten Cate, and S. Harendza, "Impact of teaching and assessment format on electrocardiogram interpretation skills," Med. Educ., vol. 44, no. 7, pp. 731-740, 2010.

A. W. Cairns et al., "A computer-human interaction model to improve the diagnostic accuracy and clinical decision-making during 12lead electrocardiogram interpretation," J. Biomed. Inform., vol. 64, pp. 93-107, 2016.

A. W. Cairns et al., "Interactive progressive-based approach to aid the human interpretation of the 12lead Electrocardiogram," in Computing in 
Cardiology Conference (CinC), 2015, pp. 197200.

[19] R. R. Bond et al., "Assessing computerized eye tracking technology for gaining insight into expert interpretation of the 12-lead electrocardiogram: an objective quantitative approach," $J$. Electrocardiol., vol. 47, no. 6, pp. 895-906, 2014.

[20] F. Heylighen, "Collective Intelligence and its Implementation on the Web: Algorithms to Develop a Collective Mental Map," Comput. Math. Organ. Theory, vol. 5, no. 3, pp. 253-280, Oct. 1999.

[21] R. R. Bond, D. D. Finlay, C. D. Nugent, and G. Moore, "A review of ECG storage formats," Int. J. Med. Inform., vol. 80, no. 10, pp. 681-697, Oct. 2011.

[22] A. Cockburn, C. Gutwin, and S. Greenberg, "A Predictive Model of Menu Performance."

[23] T. S. Jastrzembski and N. Charness, "The Model Human Processor and the Older Adult: Parameter Estimation and Validation Within a Mobile Phone Task."

[24] W. E. Hick, "Quarterly Journal of Experimental Psychology On the rate of gain of information ON THE RATE OF GAIN OF INFORMATION."

[25] R. Sassi et al., "PDF-ECG in clinical practice: A model for long-term preservation of digital 12-lead ECG data," J. Electrocardiol., 2017.

\section{Address for correspondence:}

Room 16J27, University of Ulster, Jordanstown Campus,

Shore Road, Newtownabbey, Co. Antrim, BT37 0QB

E-mail address: cairns-a3@email.ulster.ac.uk 